\title{
AVALIAÇÃO DA SUPERFÍCIE DE PAVIMENTOS FLEXÍVEIS ATRAVÉS DO LEVANTAMENTO VISUAL CONTÍNUO E ÍNDICE DE GRAVIDADE GLOBAL EXPEDITO - ESTUDO DE CASO EM TERESINA-PI
}

\author{
MELO CERQUEIRA, MARIA DE JESUS \\ Graduanda de Eng. Civil \\ Centro Universitário UNINOVAFAPI \\ Piauí; Brasil \\ mariadejesusmelocerqueira@gmail.com
}

\author{
COSTA FERREIRA, WANDERSON \\ Graduando de Eng. Civil \\ Centro Universitário UNINOVAFAPI \\ Piauí; Brasil \\ wandersoncosta.wc@gmail.com
}

\author{
MATOS DE SOUSA, ALINE \\ Graduanda de Eng. Civil \\ Centro Universitário UNINOVAFAPI \\ Piauí; Brasil \\ alivitoria84@gmail.com
}

\author{
ALVES SANTANA, CLAUDENY \\ Mestre em Engenharia de Transportes \\ Centro Universitário UNINOVAFAPI \\ Piauí; Brasil \\ cl.deny@gmail.com
}

\section{RESUMO}

Os investimentos em infraestrutura rodoviária são de grande importância para o desenvolvimento econômico de uma região, uma vez que a ausência desse investimento acarreta um número crescente de acidentes, desperdício de carga e gasto elevado com manutenção e combustíveis. O território brasileiro possui extensas rodovias, contudo, segundo dados oficiais, $60 \%$ da malha viária demonstra algum tipo de problema, sendo eles patologias características de pavimentos flexíveis. Conforme exposto neste trabalho foi analisado o desempenho funcional e realizado inspeções in loco afim de obeservar e quantificar as patologias em vias urbanas de Teresina-PI. A pesquisa aplicada tem como base exploratória, visitas no local e levantamentos quantitativos e qualitativos. Para a realização desse estudo foi feito a fundamentação teórica de acordo com a Norma DNIT 008/2003 e Norma DNIT 009/2003. O estado de conservação da via é representado através do levantamento das patologias juntamente com o auxílio dos cálculos de IGGE (Índice de Gravidade Global Expedito) e LVC (Levantamento Visual Contínuo). Por fim, de acordo com os resultados encontrados foi possível afirmar que há discrepâncias com relação ao estado de serventia da via analisada quanto a sua conservação, segurança e eficiência, além de propor maneiras convenientes de reparação.

\section{Palavras-chave: pavimentação, patologia, vias}

\section{ABSTRACT}

Investments in road infrastructure are of great importance for the economic development of a region, since the absence of this investment leads to an increasing number of accidents, waste of cargo and high expenses with maintenance and fuels. The Brazilian territory has extensive highways, however, according to official data, $60 \%$ of the road network shows some type of problem, being pathologies characteristic of flexible pavements. As shown in this paper, functional performance was analyzed and on-site inspections performed in order to observe and quantify the pathologies in urban roads of Teresina-PI. Applied research is based on exploratory, on-site visits and quantitative and qualitative surveys. For the accomplishment of this study the theoretical foundation was made according to the DNIT Standard 008/2003 and DNIT Standard 009/2003. The conservation status of the road is represented by surveying the pathologies together with the aid of IGGE (Expedited Global Gravity Index) and LVC (Continuous Visual Survey) calculations. Finally, according to the results found, it was possible to state that the discrepancies with respect to the condition of the analyzed road regarding its conservation, safety and efficiency, besides proposing convenient ways of repair.

Keywords: paving, pathology, pathways 


\section{INTRODUÇÃO}

O Sistema Viário tem como objetivo oferecer conforto e segurança durante translado de veículos independente do seu porte, assim, é necessário que as vias estejam em boas condições de uso, com boa aderência, pouco ruído e com uma condição superior de rolamento, a partir dessas características os usuários obtém uma redução no custo da manutenção de seus veículos tendo em vista que eles são diretamente ligados ao estado em que se encontra o pavimento. Os pavimentos são todas as estruturas que possuem múltiplas camadas com diferentes espessuras e materiais assentadas sobre um subleito regularizado (GOMES; SERAFIM, 2018). Entretanto, o pavimento tem como principal função resistir às ações das cargas dos veículos e melhorar as condições de rolamento, oferecendo conforto e segurança aos usuários. No Brasil existe extensas rodovias que segundo dados oficiais da CNT (Confederação Nacional do Tranportes), cerca de 60\% da malha viária apresenta algum tipo de problema devido a patologias características de pavimento flexível. Dessa forma, as patologias são classificadas como defeitos funcionais e estruturais. Após implantação de estrutura de pavimentação, o órgão responsável pela sua gestão tem por obrigação identificar e efetuar as atividades de manutenção necessárias. Essa prática é indispensável e visam manter ou restabelecer as condições da via, devendo, portanto, ser iniciadas logo após a sua abertura ao tráfego (ALVAREZ, 2018).

Segundo FEITOZA (2014), após a década de 1950, com a explosão das rodovias no Brasil, inúmeros estudos foram realizados com o intuito de otimizar a implantação e conservar de maneira eficiente os pavimentos implantados, a partir disso surgiram normas e recomendações por órgãos competentes. No entanto, no âmbito municipal, a regulamentação não é respeitada, com exceção de algumas vias estrangeiras, além disso, não existe atualização periódica das normas consolidadas o que dificulta sua aplicação em vias urbanas. O Sistema viário de Teresina, capital do Estado do Piauí, segundo o Plano Diretor apresentado em janeiro de 2008, compreende um total de $3.110 \mathrm{~km}$ (três mil cento e dez quilômetros) de extensão que cobrem uma área urbana de $283,85 \mathrm{~km}^{2}$. A cidade de Teresina é uma cidade parcialmente planejada, porém apresenta uma quantidade considerável de rua e avenidas sem pavimento ou com pavimento em péssimo estado, em muitos locais se utiliza o método "Tapa Buraco" como único método de conservação, executado de maneira desordenada, acarretando tanto desconforto quanto riscos (FEITOZA, 2014).

\section{OBJETIVO}

O presente estudo tem como objetivo principal analisar e quantificar através de inspeções in loco patologias em vias urbanas na cidade de Teresina, capital do estado do Piauí, através do método de Levantamento Visual Contínuo, além de comparar o desempenho funcional das mesmas e o conforto que proporcionam aos usuários.

\section{METODOLOGIA}

O estudo foi realizado em duas avenidas da capital do estado do Piauí. A primeira é a Av. Rui Barbosa localizada na zona norte da cidade com extensão aproximada de $7 \mathrm{~km}$ e a segunda sendo a Av. Padre Humberto Pietro Grande, localizada na zona sudeste, com extensão aproximada de $2 \mathrm{~km}$. As vias são caracterizadas como sendo do tipo flexível, entretanto, em ambas será realizado a investigação por um grupo de avaliadores que percorreram o trecho de cada via para analisar possiveis patologias.

Esse trabalho consiste em avaliar o estado de conservação dos pavimentos bem como contribuir com a definição de maneiras tecnicamente adequadas para durabilidade e/ou manutenção das vias. Para avaliar as condições superficiais do pavimento, foi feito o levantamento visual com auxílio de um veículo equipado com velocímetro/odômetro calibrado para aferição da velocidade de operação e das distâncias percorridas, sendo o mesmo operado com velocidade aproximada de $40 \mathrm{~km} / \mathrm{h}$, segundo o que diz a Norma DNIT 008/2003 - PRO.

A equipe responsável pelo levantamento foi composta por dois técnicos e o motorista do veículo. Para a avaliação subjetiva da superfície de pavimento as condições climáticas estavam favoráveis de acordo com a Norma DNIT 009/2003 - PRO. A investigação foi realizada por um grupo de avaliadores que percorreram o trechos explorados para análise do mesmo. 


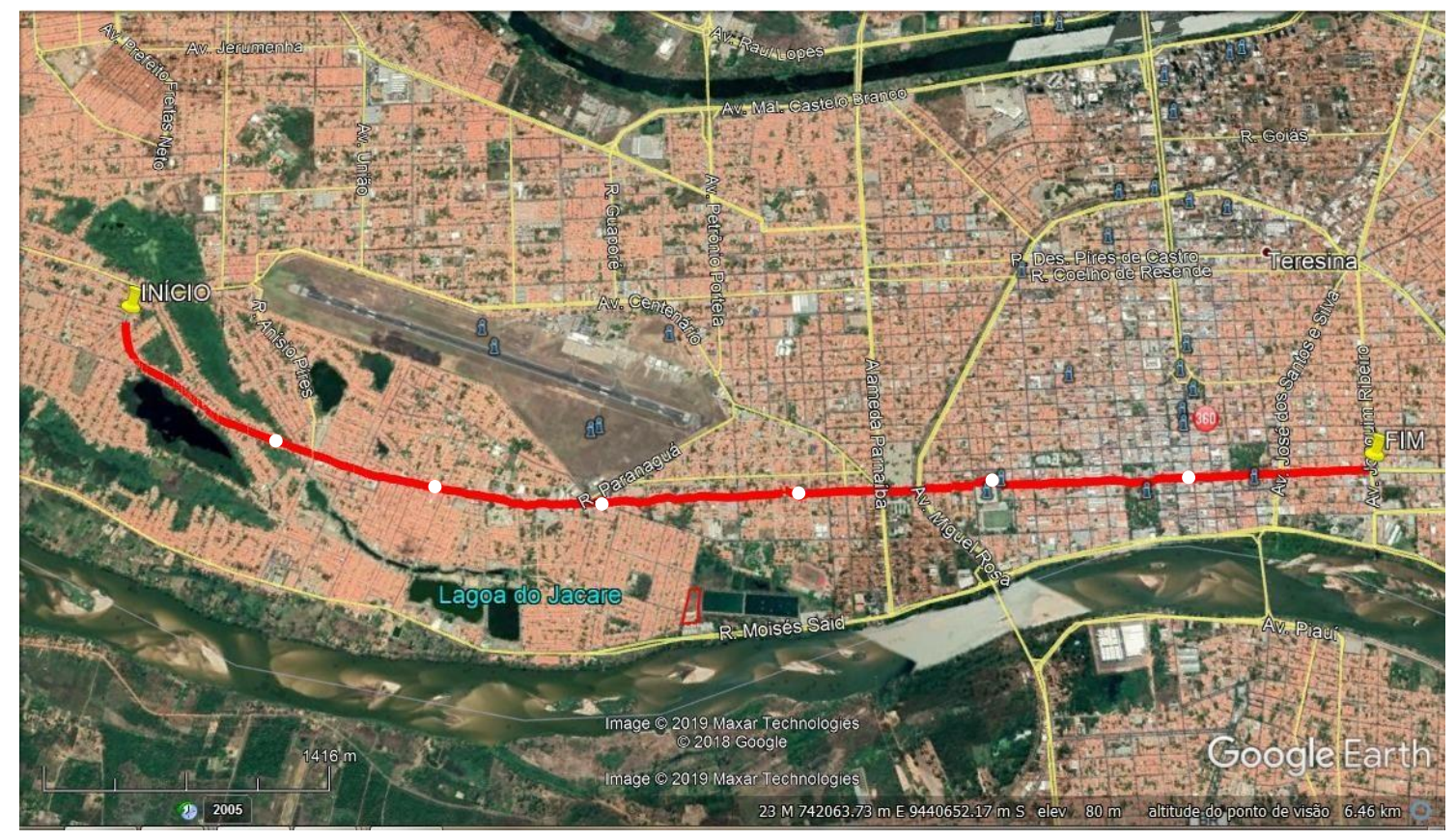

Figura 1 a) - Imagem de satélite da Avenida Rui Barbosa Fonte : Google Earth, 2020

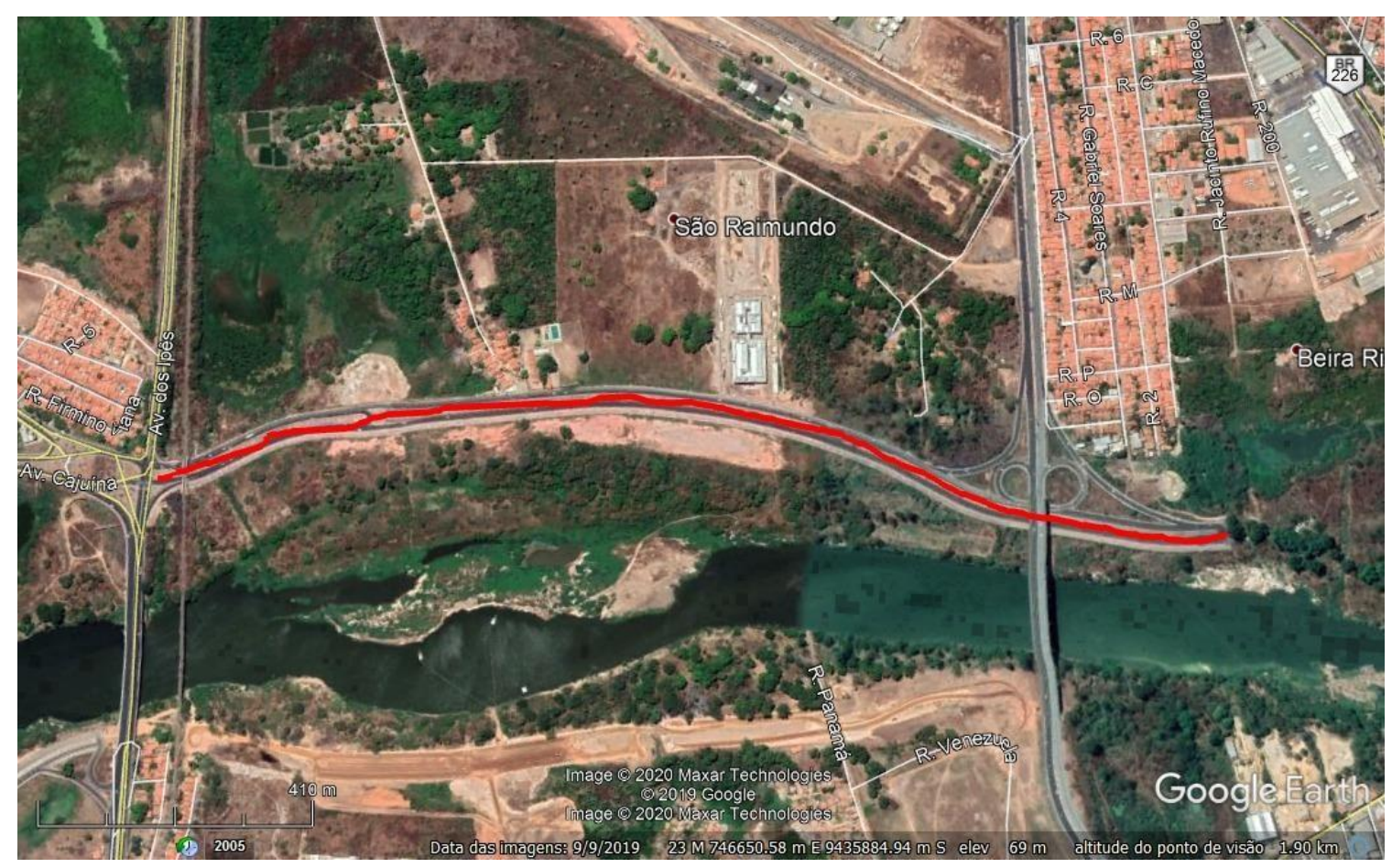

Figura 1 b) - Imagem de satélite da Avenida Padre Humberto Pietro Grande Fonte: Google Earth, 2020

\section{RESULTADOS}

Na Avenida Rui Barbosa os trechos 01 e 02 apresentaram a maior frequência de patologias, sendo classificadas como péssimas. Os defeitos mais comuns foram trincas isoladas e interligadas do tipo jacaré, causadas pela rigidez da massa asfáltica e solicitação de trafego. Também foram identificadas patologias do tipo panela ou buraco, onde são formadas pela falta de aderência entre o pavimento e camadas inferiores. Além de remendos provenientes de recuperação de panelas que foram restauradas anteriormente. 
Os trechos 03, 04 e 05, foram considerados os melhores trechos da avaliação, apresentando apenas desgaste e trincas medianas na superfície do pavimento.

Nos trechos 06 e 07 além das trincas quem são bem recorrentes em toda extensão da via, foi identificada ondulação, deformações do tipo trilho de roda, sendo essa duas causadas por instabilidade da mistura betuminosa constituintes do revestimento ou devido à fluência plástica de uma ou mais camadas do pavimento ou do subleito.

Como diagnóstico, de forma geral, a avenida apresenta um alto índice de degradação superficial em alguns trechos ao longo da área analisada. Apresentando um grande número de trincas interligadas que podem sofrer evolução, se não passarem por restauração, há também a existência de panelas que chegam a atingir a base do revestimento, que se trata de um calçamento executado em pedra lançada.

Para constatar os fatos aqui mencionados, segue as tabelas utilizadas durante o estudo em questão que comprovam os dados tidos como base na realizaçao desse estudo. A via analisada se encontra na cidade de Teresina-PI, na região central passando ainda pela zona norte, a Avenida Rui Barbosa apresenta uma extensão de 7 Km e interliga a zona norte ao centro, onde o tráfego é bem intenso. Os resultados obtidos tem como base as Normas 008/2003-PRO e 009/2003-PRO do DNIT.

Fazendo a comparação com a segunda via estudada, a Avenida Padre Humberto Pietro Grande , apresenta uma avaliaçao melhor, uma vez que por ser uma via nova, ela não apresenta uma quantidade significativa de patologias que possam comprometer o conforto do usuário, isso pode ser comprovado pelas tabelas apresentadas a seguir. As poucas patologias encontradas na via resumem-se a trincas e panelas, de baixa frequência, sendo dificultoso até quantificar essas patologias.

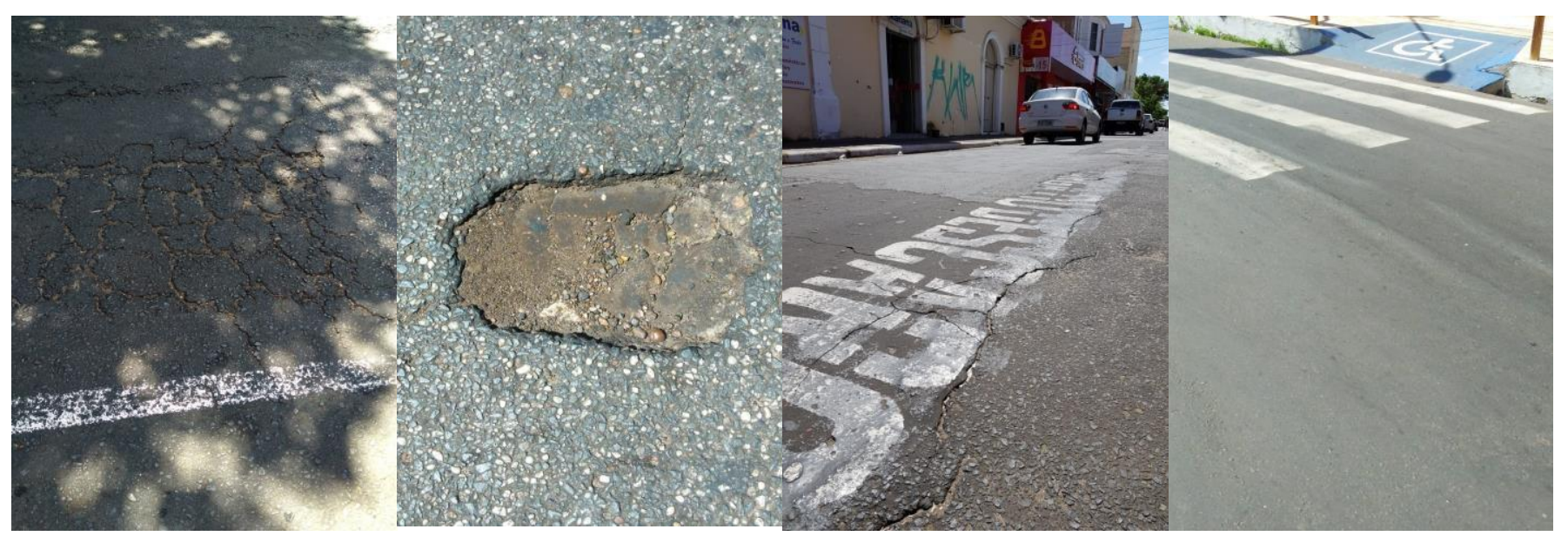

Figura 3 - Alguns tipos de patologias encontradas na Avenida Rui Barbosa em Teresina-PI

3a) Patologia couro de jacaré; 3b) Patologia panela; 3c) Patologia trincas; 3d)Patologia causada pelo sobrepeso de transportes pesados Fonte: dados da pesquisa, 2020

\subsection{LEVANTAMENTO VISUAL CONTÍNUO PARA AVALIAÇÃO DA SUPERFÍCIE DE PAVIMENTOS FLEXÍVEIS E SEMI-RÍGIDOS}

A avenidas foram divididas em trechos, a AVENIDA RUI BARBOSA em 7 trechos de $1 \mathrm{~km}$ e a AVENIDA PADRE HUMBERTO PIETRO GRANDE em 4 trechos de $0,5 \mathrm{~km}$, para garantir a uniformidade da superfície estudada. Através do LVC foi identificado os tipos de defeitos apresentados ao longo das vias. A Tabela 1 apresenta as observações feitas durante a aplicação da metodologia. 
TABELA 1 - FORMULÁRIO PARA O LEVANTAMENTO VISUAL CONTÍNUO PARA AV. RUI BARBOSA

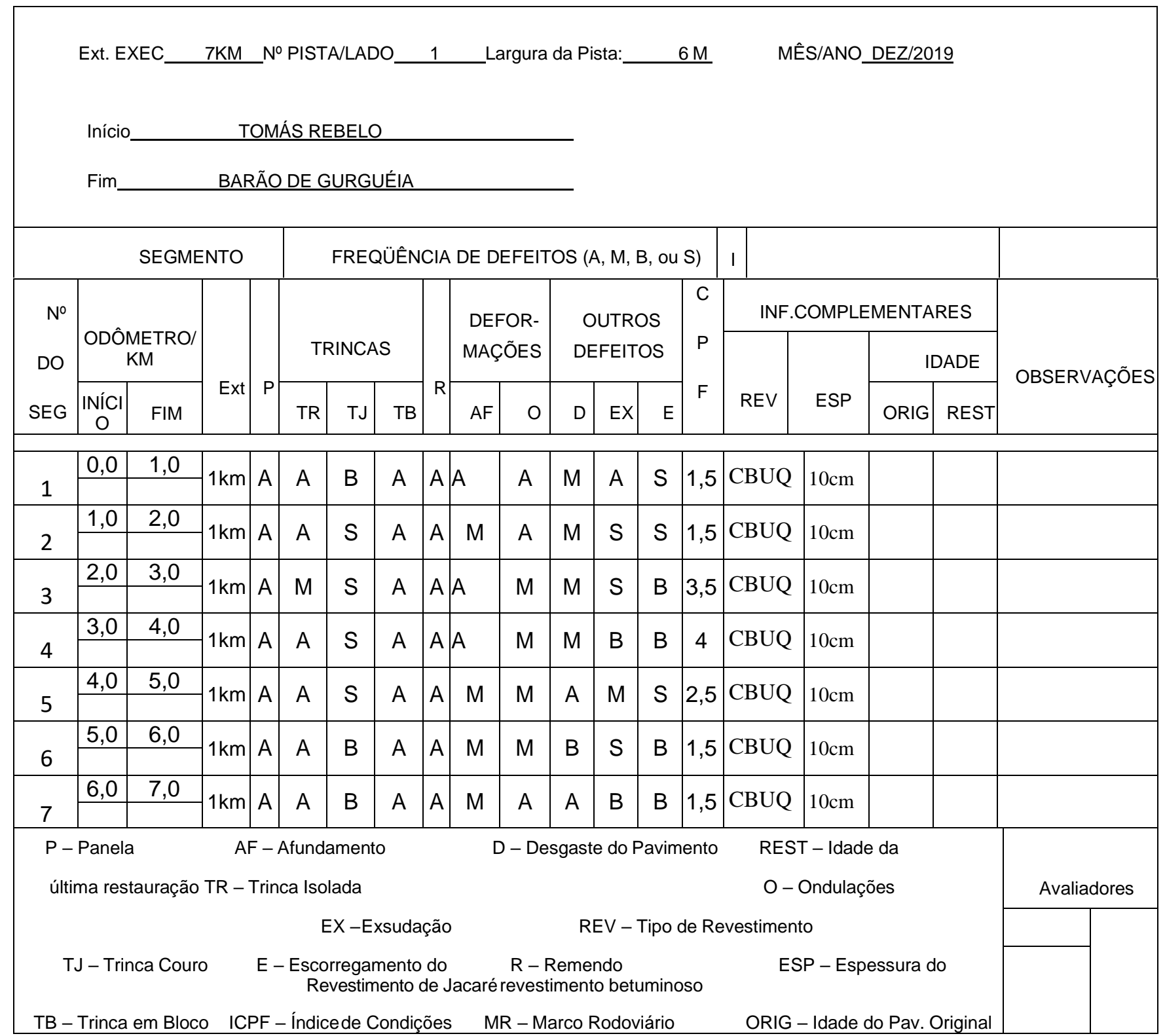

A Tabela 2 apresenta os fatores correspondentes às severidades dos defeitos anotadas pelo observador e o cálculo do Índice de Gravidade Global Expedito (IGGE) do pavimento.

O IGGE é obtido através do desenvolvimento da fórmula a seguir, tendo esta que ser feita para cada sub-trecho pesquisado, formando assim um relatório da condição do trecho pesquisado. O cálculo desse índice deve ser feito através da equação I abaixo e com o auxílio do formulário de cálculo do IGGE, segundo a Norma DNIT 008/2003 - PRO.

$$
\text { IGGE }=(\text { Pt } \times \text { Ft })+(\text { Poap } \times \text { Foap })+\left(P_{m r} \times P_{m r}\right)
$$

Onde:

$\mathrm{Ft}, \mathrm{Pt}=$ Freqüência e Peso do conjunto de trincas $\mathrm{t}$;

Foap , Poap = Frequiência e Peso do conjunto de deformações;

- Fpr, Ppr = Freqüência (quantidade por $\mathrm{km})$ e Peso do conjunto de panelas e remendos. 
TABELA 2 - CÁLCULO DO IGGE PARA AV. RUI BARBOSA

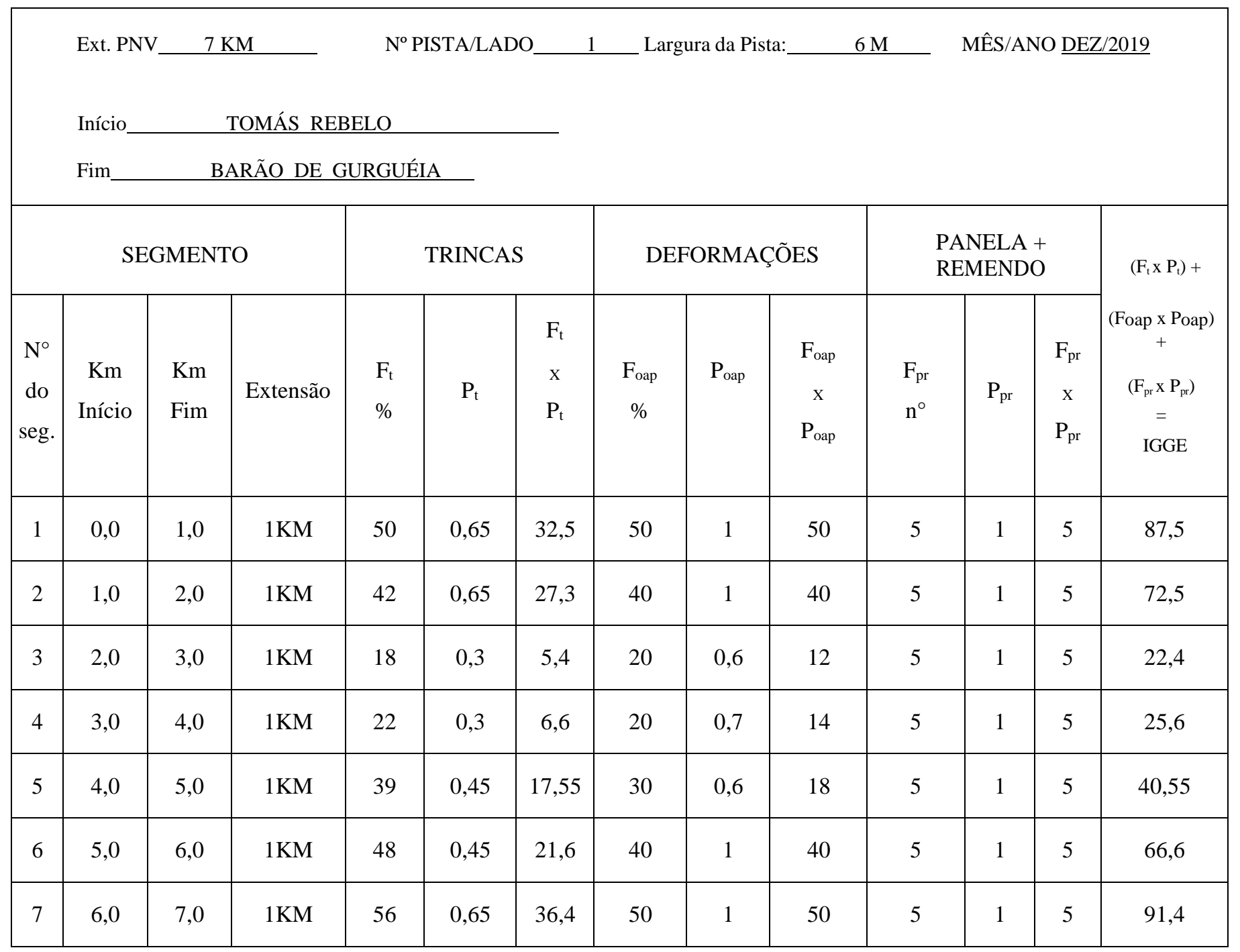


A Tabela 3 a seguir, funciona como um quadro de resumo para demonstrar a real situação da rodovia analisada e fazer uma síntese dos 03 três índices calculados anteriormente.

TABELA 3 - QUADRO RESUMO PARA AV. RUI BARBOSA

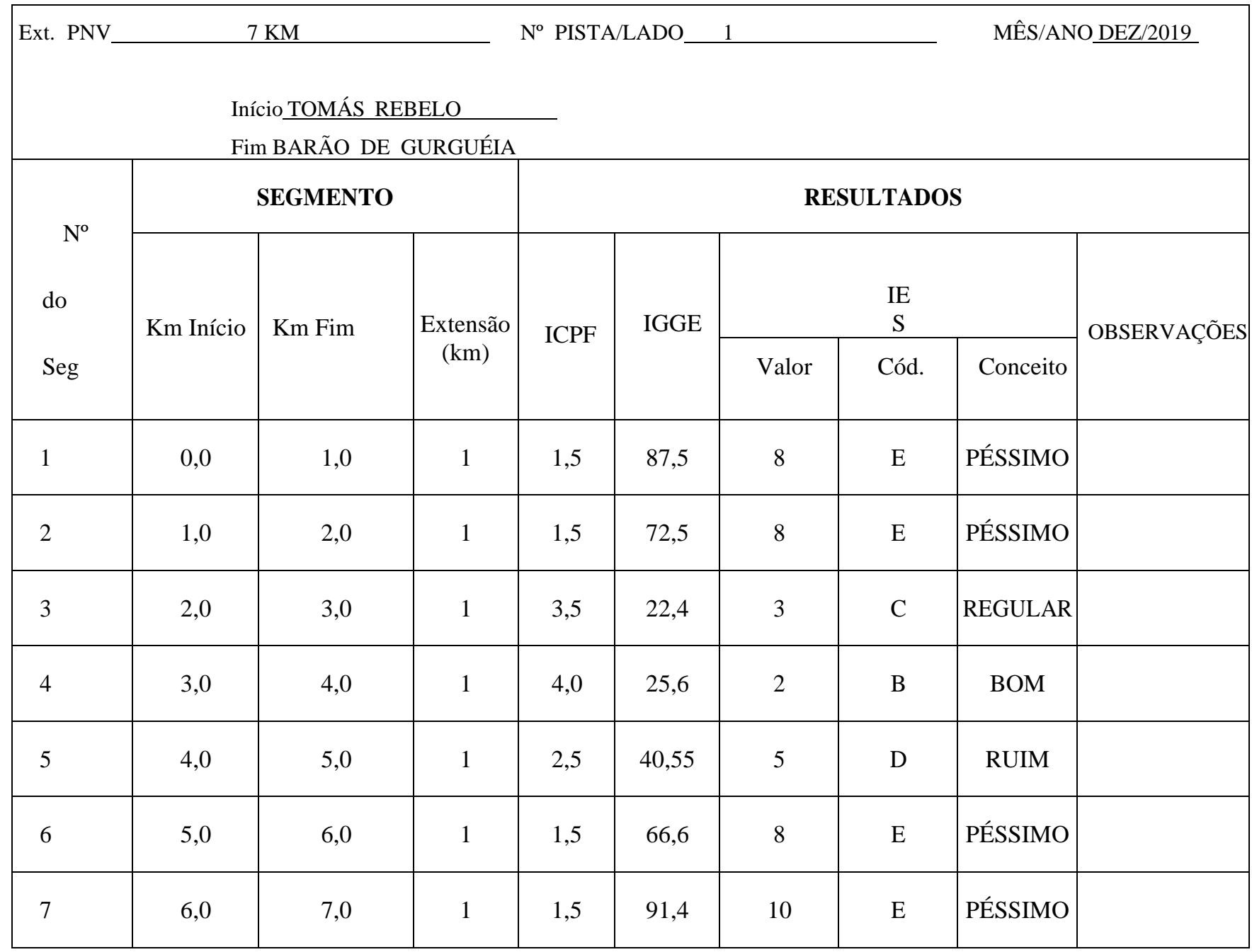

ICPF - Índice de Condição de Pavimentos Flexíveis

IGGE - Índice de Gravidade Global Expedito

IES - Índice de Estado de Superfície 
TABELA 1 - FORMULÁRIO PARA O LEVANTAMENTO VISUAL CONTÍNUO PARA AV. PADRE HUMBERTO PIETRO GRANDE

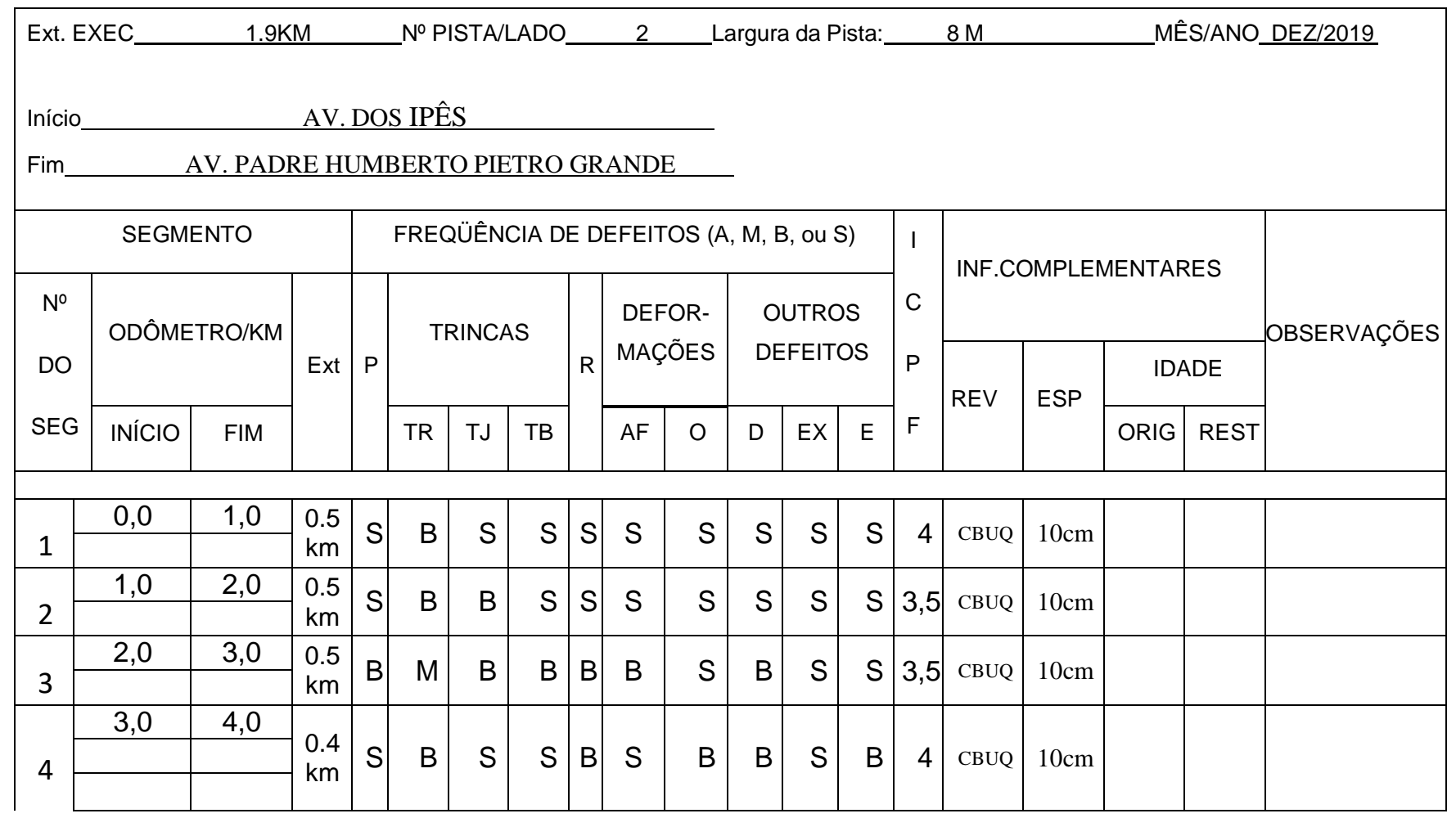

TABELA 2 - CÁLCULO DO IGGE AV. PADRE HUMBERTO PIETRO GRANDE

\begin{tabular}{|c|c|c|c|c|c|c|c|c|c|c|c|c|c|}
\hline & Ext. PNV & $7 \mathrm{KM}$ & ${ }^{\circ} \mathrm{PISTA} / \mathrm{LA}$ & & _I & sura $\mathrm{c}$ & & & & & MÊ & $\mathrm{NO}_{-}$ & $E Z / 2019$ \\
\hline & Início_ & & AV. DC & S IPÍ & & & & & & & & & \\
\hline & Fim_ & I. PAI & HUMBER & $\mathrm{O}$ PI & RO GR & & & & & & & & \\
\hline & & SEGI & NTO & & & CAS & & FOR & ÇÕES & & $\begin{array}{l}\text { NEL } \\
\text { MEN }\end{array}$ & & $(\mathrm{Ft} \times \mathrm{Pt})+$ \\
\hline $\begin{array}{l}\mathrm{N}^{\circ} \\
\text { do } \\
\text { se } \\
\text { g. }\end{array}$ & $\begin{array}{l}\text { Km } \\
\text { Início }\end{array}$ & $\begin{array}{l}\mathrm{Km} \\
\text { Fim }\end{array}$ & Extensão & $\begin{array}{l}\mathrm{F}_{\mathrm{t}} \\
\%\end{array}$ & $P_{t}$ & $\begin{array}{l}\mathrm{F}_{\mathrm{t}} \\
\mathrm{x} \\
\mathrm{P}_{\mathrm{t}}\end{array}$ & $\begin{array}{c}\mathrm{F}_{\text {oap }} \\
\%\end{array}$ & $\mathrm{P}_{\text {oap }}$ & $\begin{array}{c}\mathrm{F}_{\text {oap }} \\
\mathrm{x} \\
\mathrm{P}_{\text {oap }}\end{array}$ & $\begin{array}{l}\mathrm{F}_{\mathrm{pr}} \\
\mathrm{n}^{\circ}\end{array}$ & $\mathrm{P}_{\mathrm{pr}}$ & $\begin{array}{c}\mathrm{F}_{\mathrm{pr}} \\
\mathrm{x} \\
\mathrm{P}_{\mathrm{pr}}\end{array}$ & $\begin{array}{c}\text { (Foap x } \\
\text { Poap) + } \\
\text { (Fpr x } \\
\text { Ppr) } \\
= \\
\text { IGGE }\end{array}$ \\
\hline 1 & 0 & 1 & $0.5 \mathrm{~km}$ & 10 & 0,3 & 3 & 10 & 0,6 & 6 & 0 & 0,7 & 0 & 9 \\
\hline 2 & 1 & 2 & $0.5 \mathrm{~km}$ & 10 & 0,3 & 3 & 10 & 0,6 & 6 & 2 & 0,7 & 1,4 & 10,4 \\
\hline 3 & 2 & 3 & $0.5 \mathrm{~km}$ & 20 & 0,45 & 9 & 20 & 0,7 & 14 & 4 & 0,8 & 3,2 & 26,2 \\
\hline 4 & 3 & 4 & $0.4 \mathrm{~km}$ & 15 & 0,3 & 4,5 & 15 & 0,6 & 9 & 2 & 0,7 & 1,4 & 14,9 \\
\hline
\end{tabular}


TABELA 3 - QUADRO RESUMO AV. PADRE HUMBERTO PIETRO GRANDE

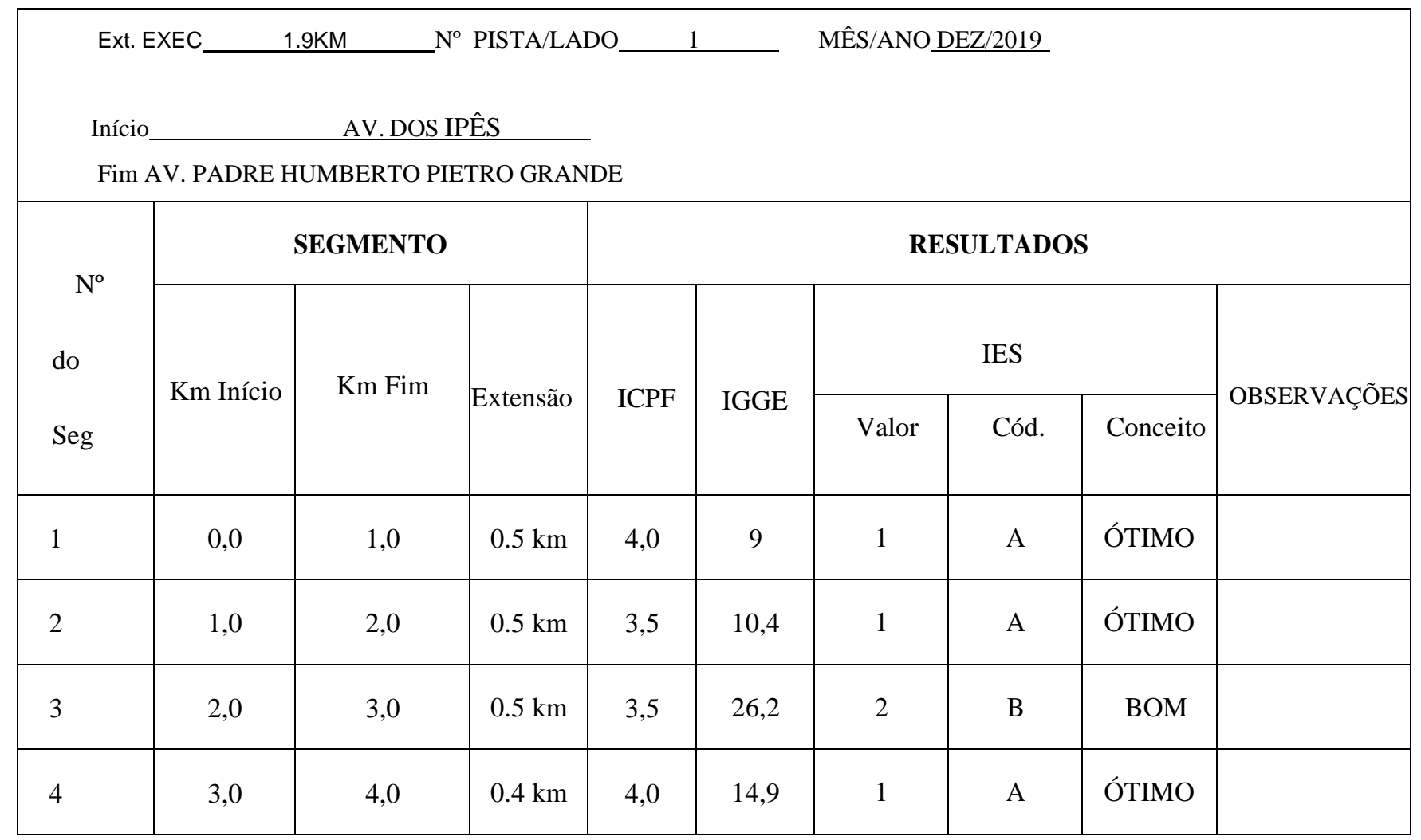

\subsection{AVALIAÇÃO SUBJETIVA DA SUPERFÍCIE DE PAVIMENTOS FLEXÍVEIS E SEMI-RÍGIDOS}

Com a realização desse procedimento foi possível ser feita a avaliação da superfície das vias estudadas, onde foram recolhidos os resultados individuais de cada um dos integrantes, no caso, seis resultados diferentes, três para cada via, porém com poucas discrepâncias, como a Norma DNIT 009/2003 prevê. O objetivo do procedimento constitui em avaliar as vias Avenida Rui Barbosa e Avenida Padre Humberto Pietro Grande quanto o que diz respeito ao Conforto e ao Valor de Serventia Atual, que deve proporcionar ao usuário uma via com rolamento suave e confortável. 
AVENIDA RUI BARBOSA

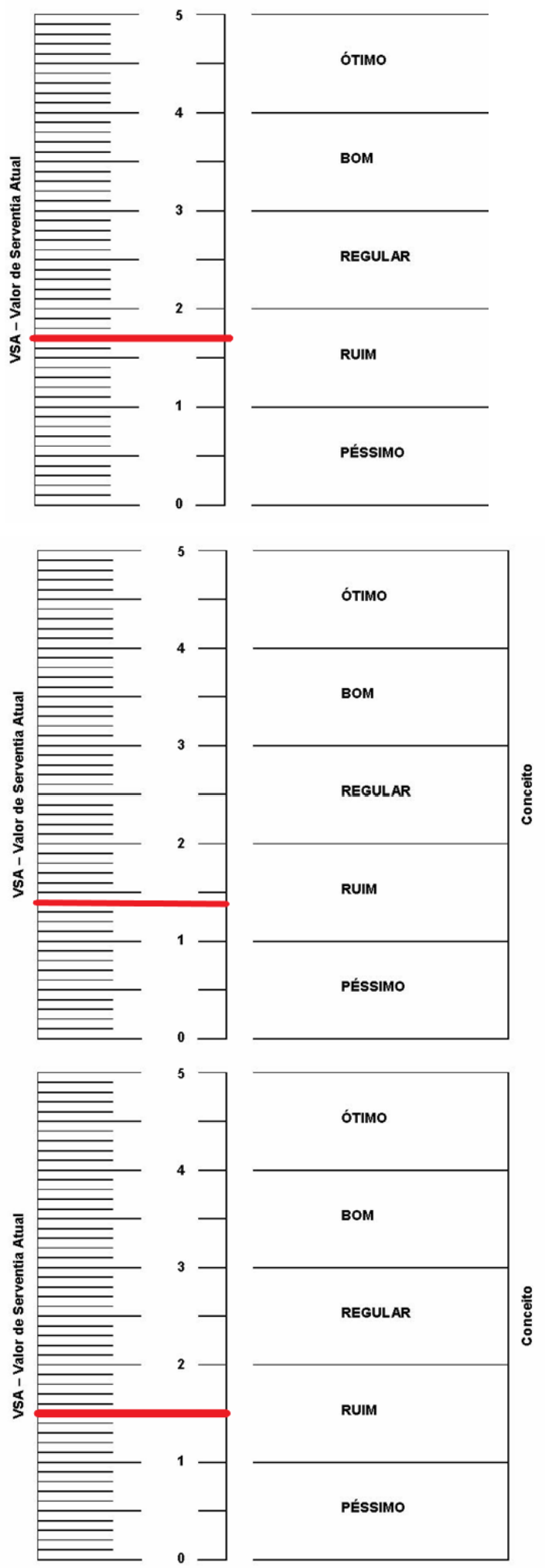

Figura 2a) - Escala de conceitos para determinação do VSA

\section{OBSERVAÇÕES 1:}

Durante o trajeto, foi possível identificar uma quantidade grande de remendos, onde o desconforto foi notado. Se analisarmos as condições que a norma estabelece, seria de grande importância que a rodovia estudada passasse por um processo de recapeamento, já que estamos analisando a possibilidade de se fazer uma viagem nessa via, como estabelece a norma.

\section{OBSERVAÇÕES 2:}

Analisando a via estudada, a presença de trincas se tornou frequente durante grande parte da via, principalmente em seu final. Foi possível notar também que as trincas originaram panelas. Além de trincas, a patologia conhecida como afundamento também era presente na via.

\section{OBSERVAÇÕES 3:}

No decorrer da nossa análise, percebemos uma boa quantidade de patologias presentes na Avenida Rui Barbosa que contribuem significativamente para o desconforto da mesma. Dentre as patologias, foi observado que as trincas eram muito constantes, havendo também a desagregação do pavimento em uma pequena área de um dos trechos. 
AVENIDA PADRE HUMBERTO PIETRO GRANDE
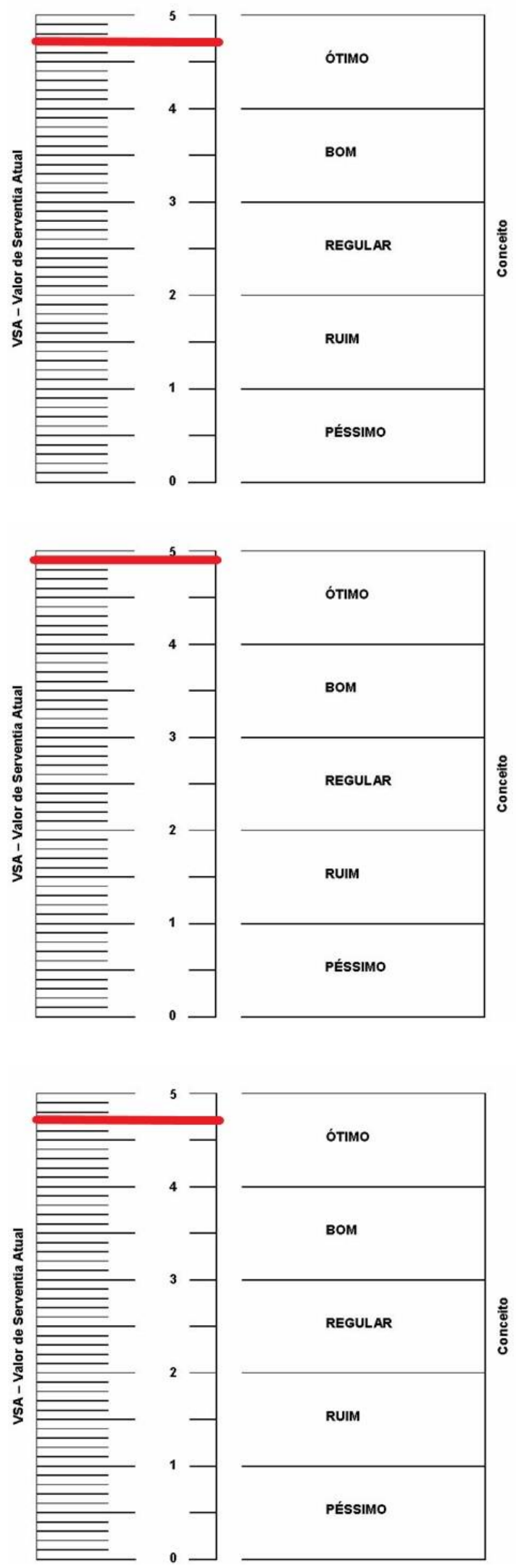

Figura 2b) - Escala de conceitos para determinação do VSA

\section{OBSERVAÇÕES 1:}

Na via em questão pode-se visualizar a presença de poucas patologias, ou seja, a pavimentação se encontra em bom estado apresentando apenas desgastes superficiais e trincas não muito severas, dessa forma ela oferece boas condições de utilização e conforto para o usuário.

\section{OBSERVAÇÕES 2:}

A via analisada apresentou um bom estado de conservação, foram identificadas poucas patologias no decorrer do percurso, sendo encontrada apenas uma "panela" (buraco) e um escoamento de revestimento em ponto específico. Também foram encontradas algumas trincas, com isso, pode ser considerada segura e de boa comodidade para os veículos.

\section{OBSERVAÇÕES 3:}

Após análise do percurso, foi possível concluir que a via em questão é considerada ótima, apresentando poucas patologias, dentre eles as trincas sãs as mais comuns como também a presença de uma panela, não comprometendo em nada o conforto no decorrer do percurso. Deve-se levar em consideração que essas especificações podem ter tido um bom resultado devido a via ser nova e, portanto, não ter tido um grande tráfego de veículos como a primeira e nem ter passado por intempéries tanto com a Rui Barbosa. 
O Valor de Serventia Atual (VSA) da AVENIDA RUI BARBOSA adotado depois de feita a média aritmética dos integrantes foi de 1,7, ainda considerado como RUIM. Já o VSA da AVENIDA PADRE HUMBERTO PIETRO GRANDE foi de 4,77, sendo considerado ótimo e propício ao conforto do usuário.

\section{CONCLUSÃO}

Em alguns trechos, pode-se constatar alguns tipos de patologias como fissuras que são frequentes nas duas vias estudadas, tornando visível que em alguns trechos determinadas patologias são mais evidentes que em outras e que isso influi diretamente no que diz respeito ao conforto do usuário que utiliza a via como acesso durante o seu dia-a-dia.

De acordo com o descrito ao longo deste trabalho evidencia-se que é de grande importância o conhecimento dos tipos de defeitos dos pavimentos para se constituir uma análise satisfatória das condições dos mesmos e para desenvolvimento do gerenciamento dos pavimentos com intuitos técnicos e econômicos, além de direcionar as melhores técnicas de recuperação.

Com base no levantamento feito, pode se concluir que a Avenida Padre Humberto Pietro Grande em sua maior parte apresenta um estado de conservação que varia de regular a bom. O trecho apresenta defeitos diversos, porém, com severidades medianas, a não ser em trechos específicos que apresentaram falhas mais severas. Já na avenida Rui Barbosa, os índices de serventia encontrados, para os trechos estudados, se classifica como ruim, elucidando a importância que a rodovia estudada passasse por um processo de recapeamento. Desta forma notou-se que ambas as vias necessitam de manutenção, demandando primeiramente uma análise mais detalhada das patologias em toda sua extensão.

\section{REFERÊNCIAS}

ALVAREZ, Pâmela Suelen; RODGHER, Sandra Fabiana. PATOLOGIAS E MANUTENÇÃO NO PAVIMENTO ASFÁLTICO NO MUNICÍPIO DE BOCAINA/SP. 2018. 13 f. TCC (Graduação) - Curso de Engenharia Civil, Universidade de Araraquara, São Paulo, 2018.

Departamento Nacional de Infraestrutura de Transportes (2003). Levantamento visual contínuo para a avaliação da superfície de pavimentos flexíveis e semi-rígidos. Rio de Janeiro, DNIT - 008/2003 - PRO.

Departamento Nacional de Infraestrutura de Transportes (2003). Avaliação subjetiva da superfície de pavimentos flexíveis e semi-rígidos - Procedimento. Rio de Janeiro, DNIT - 009/2003 -PRO.

FEITOZA, E. C. Análise patológica e técnicas de recuperação de pavimentos flexíveis em vias urbanas. $2014.56 \mathrm{f}$. TCC (Graduação) - Curso de Engenharia Civil, Universidade Federal do Piauí, Teresina, 2014.

GOMES I. P.; SERAFIM L. A. Estudo Do Desempenho Funcional da Rodovia MG/329 e Propostas de Soluções para as Patologias Identificadas. 2018. 60f. TCC (graduação)- Curso de Engenharia Civil, Faculdades Doctum de Caratinga, 2018. 